\title{
The use of the double-balloon enteroscope for endoscopic retrograde cholangiopancreatography and biliary stent placement after Roux-en-Y hepaticojejunostomy
}

RouX-en-Y biliodigestive reconstruction excludes the biliary tract from conventional endoscopic retrograde cholangiopancreatography (ERCP) [1 - 3]. However, the 200-cm-long double-balloon enteroscope (DBE) facilitates intubation of the afferent Roux-en-Y limb and maintains its position in front of the biliodigestive anastomosis. The $2.8-\mathrm{mm}$ working channel allows for the introduction of conventional accessories, provided they have $230 \mathrm{~cm}$ length available. Choledochojejunostomy balloon dilation using DBE was first reported after living donor liver transplantation [4]. In one multicenter study, two patients successfully underwent DBE ERCP and biliary balloon dilation after Roux-en-Y reconstruction [5].

We report two DBE interventions in one patient who had developed a hepaticojejunostomy stricture after resection of the right lobe of the liver. This 72-year-old man with a Klatskin IIIa tumor underwent curative right hepatectomy and Roux-en-Y hepaticojejunostomy in 2004. Two years later, he presented with cholangitis and dilatation of the intrahepatic biliary tree in the remaining left liver without any evidence of tumor recurrence on magnetic resonance imaging. With the patient under general anesthesia, we reached the hepaticojejunostomy using the Fujinon EN-450T5 therapeutic DBE (Fujinon Corporation, Saitama-Shi, Saitama, Japan). The anastomosis was impacted with sludge entrapped in remaining sutures ( Figure 1). It was cannulated with a sclerosing needle catheter to perform cholangiography ( $\bullet$ Figure 2 ). The anastomotic stricture was dilated up to $6 \mathrm{~mm}$ using an esophageal dilation balloon, leading to swift clearance of intrahepatic contrast ( $\bullet$ Figure 3 ). The cholangitis recurred 5 months later as a result of occlusion of the hepaticojejunostomy. During a second DBE procedure we created a new orificium with a sclerosing needle ( Figure 4). The anastomosis was dilated over a guide wire, and two 7Fr biliary stents $(12 \mathrm{~cm}$ and $9 \mathrm{~cm})$ were introduced through the DBE into the left hepatic branch to avoid rapid stenosis $(\diamond$ Figure 5, 6). We used a nasobiliary

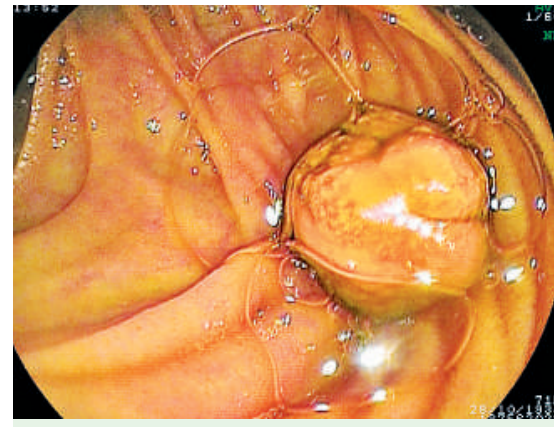

Figure 1 Endoscopic view of the hepaticojejunostomy during the first procedure showing sludge impacted in the remaining sutures.

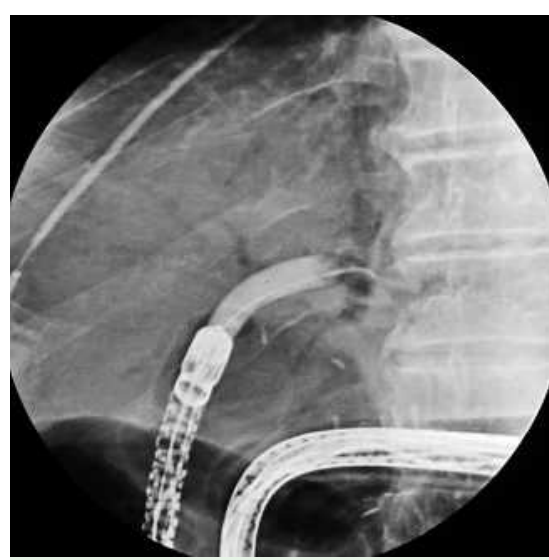

Figure 2 Radiologic view of balloon dilation of the hepaticojejunostomy up to $6 \mathrm{~mm}$ using a conventional esophageal dilation balloon. The balloon is filled with diluted iodinated contrast material (procedure 1).

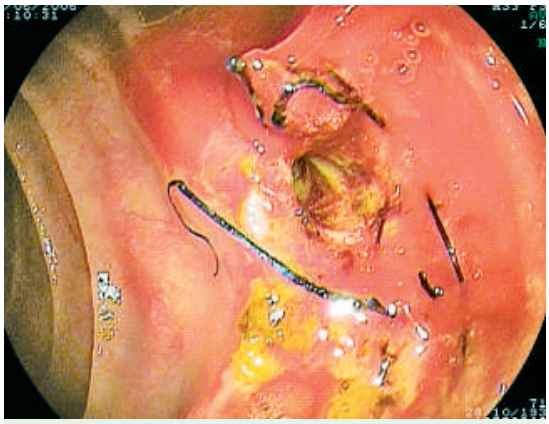

Figure 3 Endoscopic view of the dilated hepaticojejunostomy at the end of the first procedure. Note the remaining surgical sutures.

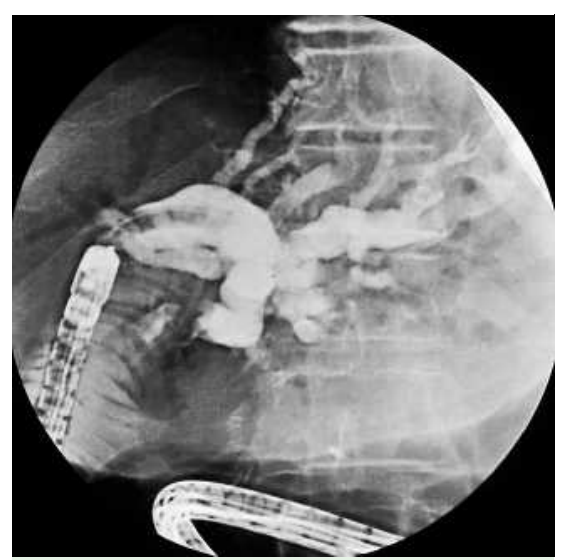

Figure 4 Radiologic view of the cholangiography after a new access to the dilated left hepatic branch had been created using a sclerosing needle. The cholangiography was performed with a dilation balloon in place (procedure 2).

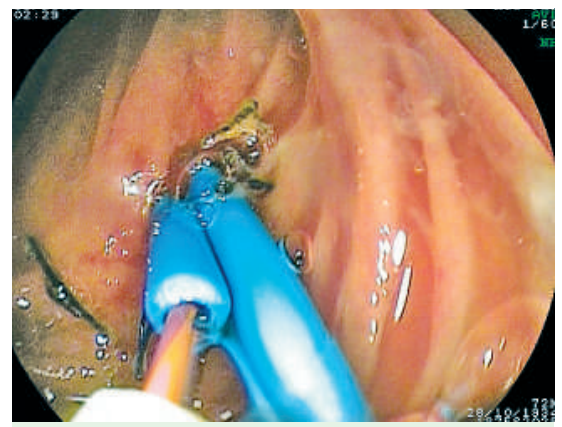

Figure 5 Endoscopic view of biliary stent (7 $\mathrm{Fr}$ ) placement over a guide wire, using a nasobiliary catheter as a pushing device. The first biliary stent is already in position (procedure 2).

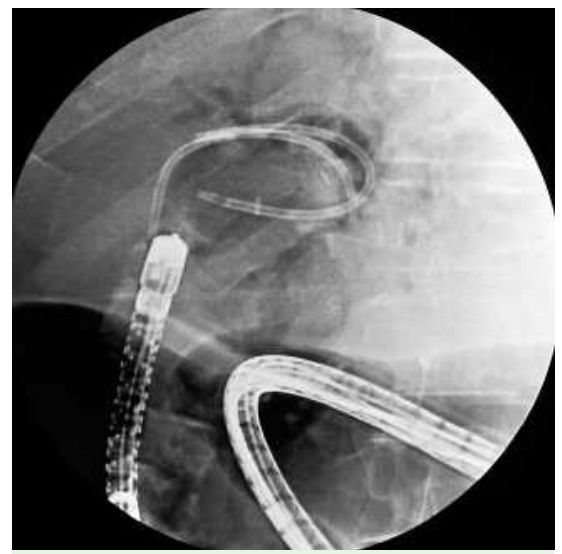

Figure 6 Radiologic view of the two biliary stents located in the hepaticojejunostomy. Note the air cholangiography at the end of procedure 2 . 
catheter as a pushing device to introduce the stents.

Evidence is emerging that DBE allows ERCP interventions after Roux-en-Y surgery. Although it remains an elaborate procedure, it might prevent the need for redo surgery. Accessories adapted to the therapeutic DBE should be made available to enable further developments for this new indication.

Endoscopy_UCTN_Code_TTT_1AP_2AD

Endoscopy_UCTN_Code_TTT_1AR_2AG

Endoscopy_UCTN_Code_TTT_1AR_2AK

\section{T. G. Moreels ${ }^{1}$, B. Roth ${ }^{1}$, E. J. Vander-} vliet $^{2}$, P. M. Parizel ${ }^{2}$, J. Dutré ${ }^{3}$,

P. A. Pelckmans ${ }^{1}$

1 Department of Gastroenterology and Hepatology, Antwerp University Hospital, Antwerp, Belgium

2 Department of Radiology, Antwerp University Hospital, Antwerp, Belgium

3 Department of Gastroenterology and Hepatology, ZNA Jan Palfijn Hospital, Merksem, Belgium

\section{References}

1 Valera-Sanchez Z, Flores-Cortes $M$, RomeroVargas ME et al. Biliodigestive anastomosis in liver transplantation: review of 13 years. Transplant Proc 2006; 38: 2471 - 2472

2 Yazumi S, Chiba T. Biliary complications after a right-lobe living donor liver transplantation. J Gastroenterol 2005; 40: 861 865

3 Saad WE, Davies MG, Saad NE et al. Transhepatic dilation of anastomotic biliary strictures in liver transplant recipients with use of a combined cutting and conventional balloon protocol: technical safety and efficacy. J Vasc Interv Radiol 2006; 17: 837 843

4 Haruta $H$, Yamamoto $H$, Mizuta $K$ et al. A case of successful enteroscopic balloon dilation for late anastomotic stricture of choledochojejunostomy after living donor liver transplantation. Liver Transpl 2005; 11 : $1608-1610$

5 Mehdizadeh S, Ross A, Gerson L et al. What is the learning curve associated with doubleballoon enteroscopy? Technical details and early experience in 6 US tertiary care centers. Gastrointest Endosc 2006; 64: 740 750
Bibliography

DOI 10.1055/s-2007-966410

Endoscopy 2007; 39: E196-E197

(c) Georg Thieme Verlag KG Stuttgart · New York . ISSN 0013-726X

\section{Corresponding author}

\section{T. G. Moreels, MD, PhD}

Antwerp University Hospital

Department of Gastroenterology and Hepatology Wilrijkstraat 10

B-2650 Antwerp

Belgium

Fax: +32-38214478

tom.moreels@uza.be 